\title{
Wirksamkeit von antipsychotischer Kombinationstherapie bei Schizophrenie
}

Fragestellung: Wir wirksam ist eine Kombinationstherapie bei Schizophrenie, wenn Patienten auf ein Antipsychotikum nicht ausreichend ansprechen?

Hintergrund: Es liegt eine sehr gute Datenlage zur Wirksamkeit von Antipsychotika bei Schizophrenie vor, wobei Amisulprid, Risperidon und Olanzapin die größten Effektstärken aufweisen. Da aber viele Patienten nicht ausreichend auf das erste Antipsychotikum ansprechen, werden häufig zwei oder gar mehr Antipsychotika oder diese mit anderen Substanzgruppen miteinander kombiniert. Die vorliegende Meta-Metaanalyse untersuchte die Wirksamkeit von 42 Medikamentenkombinationen.

Methodik: Die Autoren führten eine Metaanalyse von $29 \mathrm{Me}$ taanalysen durch, die vor dem 13. Mai 2016 publiziert worden waren. Die Daten wurden nach Standardmethoden extrahiert und es wurden Effektstärken für einzelne Antipsychotikakombinationen oder die Kombination von Antipsychotika mit nicht antipsychotischen Medikamenten gegenüber Placebobehandlung oder einer antipsycho-

Correll CU, Rubio JM, InczedyFarkas $\mathrm{G}$ et al. Efficacy of 42 pharmacologic cotreatment strategies added to antipsychotic monotherapy in schizophrenia. JAMA Psychiatry 2017; 74: $675-84$ tischen Monotherapie bei Erwachsenen mit Schizophrenie kalkuliert. Der primäre Outcome war die allgemeine Symptomreduktion. Die wichtigsten sekundären Outcomes waren die Reduktion von positiven und negativen
Symptomen, kognitiven und depressiven Symptomen sowie das Absetzen der Medikation.

Ergebnisse: 29 Metaanalysen, die insgesamt 42 Kombinationstherapien in 381 randomisiert kontrollierten Studien mit knapp 20.000 schizophrenen Patienten untersucht hatten, wurden in die Meta-Metaanalyse eingeschlossen. 14 Kombinationstherapien waren den Kontrollbehandlungen mit Effektstärken von 1,27 bis 0,23 signifikant überlegen. Die Kombinationen eines Antipsychotikums mit einem Antidepressivum waren wirksamer als die mit einem zweiten Antipsychotikum. Dazu gehörten insbesondere die stark überlegenen Kombinationen aus Antipsychotikum mit SNRI $(1,27)$, mit Lamotrigin $(0,73)$ und mit Mirtazapin $(0,72)$, sowie die mittelwirksamen mit Lithium $(0,63)$ und Topiramat $(0,58)$. Die Kombination eines Antipsychotikums mit SSRI, trizyklischen Antidepressiva, Valproinsäure oder Carbamazepin war dagegen nicht besser wirksam als die Monotherapie. Im Fall von Clozapin war keine der Kombinationstherapien der alleinigen Clozapin-Behandlung überlegen. Die Qualität der Metaanalysen war durchweg hoch, jedoch war die Qualität der eingeschlossenen randomisiert kontrollierten Studien häufig niedrig. Studien mit niedriger Qualität zeigten größere Effektstärken als Studien mit hoher Qualität.

Schlussfolgerungen: Die Autoren sehen mehrere Kombinationsbehandlungen als möglicherweise empfehlbar an, wobei aufgrund der häufig niedrigen Studienqualität die Daten mit Vorsicht zu interpretieren sind.

\section{Kombinationen aus zwei Antipsychotika nicht empfehlenswert}

Die von der sehr erfahrenen Autorengruppe vorgelegte Metaanalyse über eine große Anzahl von Metaanalysen bringt mehr Klarheit in den möglichen Nutzen von AntipsychotikaKombinationstherapien. Interessant ist, dass Patienten, die nicht auf ein Antipsychotikum allein ansprechen, bezüglich der Gesamtsymptomatik offenbar besser von einer Kombination mit einem Antidepressivum als mit einem zweiten Antipsychotikum profitieren. Das widerspricht deutlich dem gängigen Tun in vielen Kliniken, obwohl auch internationale Leitlinien immer eine Mono- vor einer Kombinationstherapie von Antipsychotika empfehlen. Bei den Antidepressiva scheint die kombinierte Aktivierung des serotonergen und noradrenergen Systems entscheidend zu sein, da zusätzliche SSRI weniger wirksam sind. Und die Blockade der Serotonin-3-Rezeptoren ist wirksam, ein Prinzip, das sich auch im Mirtazapin wiederfindet. Dazu passt, dass auch selektive Serotonin-3-Rezeptor-Blocker als add on stark wirksam sind. Bisher hat kli- nisch die Angst dominiert, die psychotische Symptomatik könnte durch die zusätzliche Gabe von Antidepressiva exazerbieren. Dies scheint einer aktuellen Studie nach nicht der Fall zu sein [1]. Wenn man den Effekt auf positive und negative Symptome differenziert betrachtet, fällt auf, dass Mirtazapin bei persistierenden positiven Symptomen eher besser zu sein scheint, während SNRI und Lamotrigin eher auf negative Symptome wirken. Dies kann klinisch direkt umgesetzt werden. Es bleibt nur die insgesamt vorsichtige Interpretation, da die in den Metaanalysen eingeschlossenen Studien häufig von methodisch schlechter Qualität waren. Hier zeigte sich wieder einmal, dass schlechte Studienqualität mit größeren Effekten assoziiert ist und dass die Effektstärken mit zunehmender Zahl hochwertiger Studien kleiner werden.

\footnotetext{
Referenz

1. Helfer B et al. Am J Psychiatry 2016; 173: 876-86
} 